\title{
Warum wir an die Italiener denken sollten
}

\author{
Bettina Maeschlia, Erminia Gagliottaa, Philip Bruggmann ${ }^{a, b}$ \\ a Hepatitis Schweiz, Zürich; ${ }^{\text {b }}$ Arud Zentrum für Suchtmedizin, Zürich
}

Italienerinnen und Italiener in der Schweiz, die über 60 Jahre alt sind, sind besonders häufig von Hepatitis $C$ betroffen. Angesteckt haben sie sich meist in den 50er und 60er Jahren im paramedizinischen Setting in ihrem Heimatland. Da eine chronische Hepatitis $C$ heute unkompliziert heilbar ist, lohnt sich der Test bei dieser Bevölkerungsgruppe.

L.F. ist als junger Mann aus Sizilien in die Schweiz gezogen. Bei einem ärztlichen Routine-Check-up im Jahr 2006 wird bei ihm Hepatitis C diagnostiziert. Die Diagnose erreicht ihn völlig unvorbereitet, da er keinerlei Beschwerden hat. Neben den regelmässigen Kontrollen, bestehend aus Abdomensonografie, Fibroscan und Bluttest schlagen ihm die Ärzte eine Therapie mit Interferon vor, die er aber aufgrund der belastenden Nebenwirkungen und des asymptomatischen Verlaufs seiner Krankheit ablehnt. Erst 2020 unterzieht sich der Patient einer Therapie. Diesmal mit den seit wenigen Jahren neu zugelassenen antiviralen Medikamenten. Die Therapie sieht die Einnahme einer einzigen Tablette pro Tag vor, dauert gesamthaft 84 Tage und weist praktisch keine Begleiterscheinung auf. L.F. ist bereits unter Therapie virenfrei und bleibt es bis heute. Mit der Heilung von Hepatitis $C$ konnte bei ihm zu seiner grossen Erleichterung das Risiko für Folgeerkrankungen beseitigt werden.

\section{Italiener vier Mal häufiger betroffen}

Die Geschichte des heute 76-jährigen Süditalieners ist nicht untypisch. In der Schweiz leben ungefähr 32000 Menschen mit Hepatitis C. Laut einer Auswertung der Daten in der schweizerischen Hepatitis-C-Kohorte und dem obligatorischen Meldewesen des Bundesamtes für Gesundheit BAG sind in Italien geborene Menschen über 60, die in der Schweiz wohnen, fast vier Mal häufiger von Hepatitis $\mathrm{C}$ betroffen als der Rest der Schweizer Bevölkerung [1].

Der Hintergrund sind Vitaminkuren und andere paramedizinische Praktiken in Süditalien in den 50er und $60 e r$ Jahren. Wenig geschultes Personal hat damals in italienischen Dörfern die ganze Bevölkerung mit Injektionen behandelt, wobei nicht selten dieselben, ungenügend sterilisierten Glasspritzen benutzt wurden [2-4].

\section{Hepatitis $\mathrm{C}$ ist heute heilbar}

In den letzten Jahren wurde die Hepatitis-C-Therapie revolutioniert. Lange waren interferonbasierte Therapien kombiniert mit Ribavirin die einzige Behandlungsmöglichkeit für eine chronische Hepatitis-C-Infektion. Da diese Therapien langwierig waren, bis zu einem Jahr oder länger dauerten und teilweise sehr schwere Nebenwirkungen nach sich zogen, wurden nur Patientinnen und Patienten mit fortgeschrittenen Leberschäden behandelt.

2014 kamen die ersten Direct Acting Antivirals Agents (DAAs) auf den Markt. Zuerst mussten diese noch zusammen mit Interferon verabreicht werden. Heute sind Kombinationstherapien Standard, die ohne Interferon auskommen. Eingenommen werden eine oder drei Tabletten pro Tag, während acht oder zwölf Wochen. Die Heilungsraten betragen über 96 Prozent.

Mit den neuen Therapien haben sich auch die Behandlungsempfehlungen geändert: Heute sollte allen chronisch mit Hepatitis C Infizierten eine Therapie angeboten werden [5]. Eine Hepatitis-C-Infektion schreitet über Jahre und Jahrzehnte meist nur langsam voran und führt in 5 bis 20 Prozent der Fälle zu einer Leberzirrhose. 2-4 Prozent der Patientinnen und Patienten mit einer Zirrhose entwickeln pro Jahr ein hepatozelluläres Karzinom (HCC). Hepatitis C ist auch heute noch eine der häufigsten Ursachen für HCC sowie ein häufiger Grund für Lebertransplantationen. Einerseits kön- 


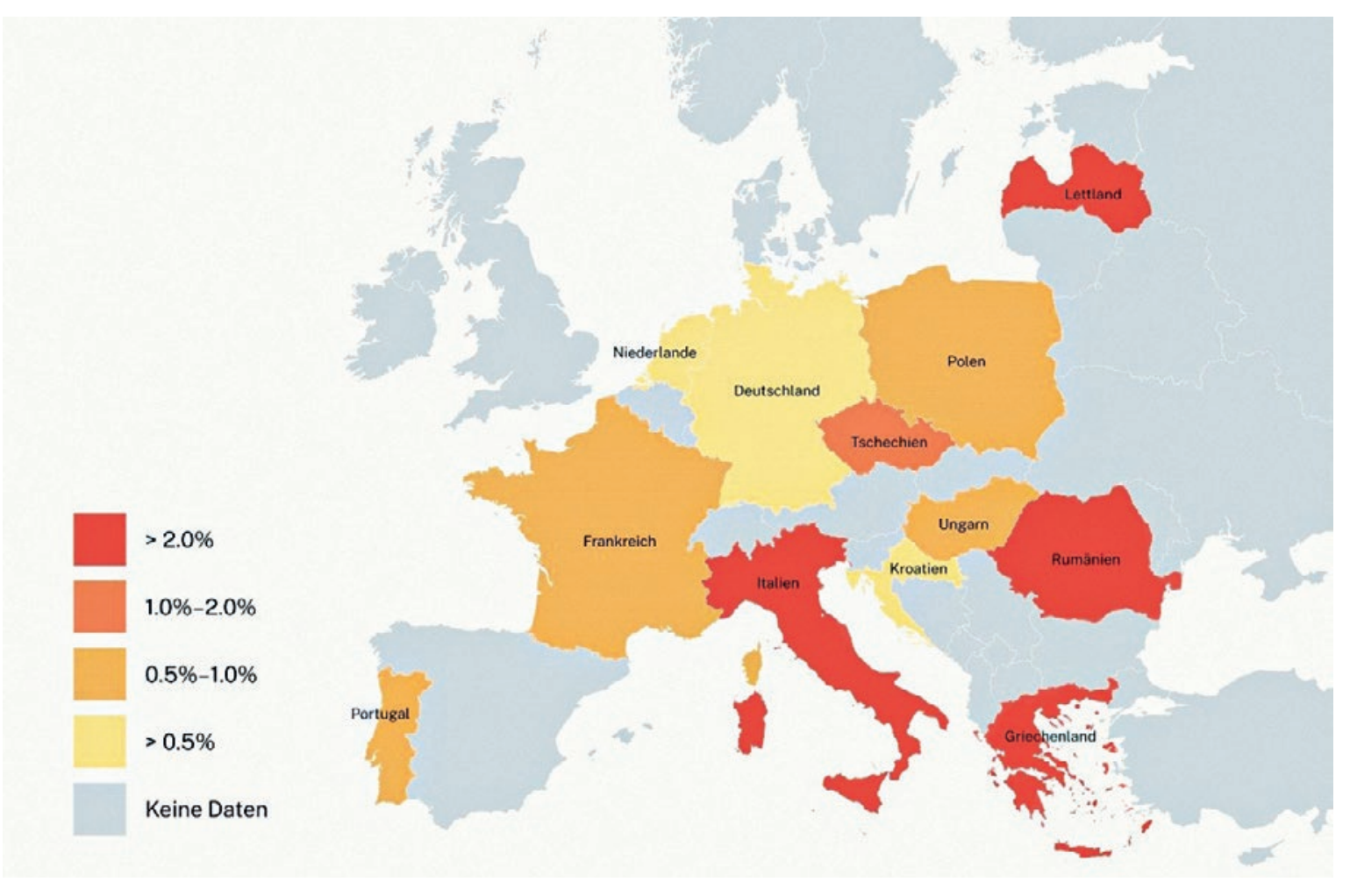

Hepatitis-C-Prävalenz in Europa (adaptiert von: ECDC Technical Report: Prevention of hepatitis B and C in the EU/EEA and the UK, November 2020 - Copyright Hepatitis Schweiz).

nen dank der neuen hocheffizienten Behandlungsmöglichkeiten Leberkrebs und Transplantationen verhindert werden, andererseits verursacht Hepatitis C eine Vielzahl weiterer Erkrankungen ausserhalb der Leber, die mit einer rechtzeitigen Therapie verhindert werden können. Dazu gehören etwa Diabetes oder Herz-Kreislauf-Erkrankungen sowie Lymphome. Zudem klagt ein grosser Teil der Patientinnen und Patienten über starke Müdigkeit, die in einigen Fällen auch zur Arbeitsunfähigkeit führen kann [6].

\section{Behandlung auch in der Hausarztpraxis}

Auch aus Public-Health-Sicht ist es angezeigt, eine chronische Hepatitis C, die wie im Falle von L.F. oft asymptomatisch ist oder ohne spezifische Symptome begleitet wird, zu diagnostizieren und zu behandeln. Dank der Therapien und dank der nur wenigen Neuansteckungen wäre es heute möglich, Hepatitis C zu eliminieren.

Die DAAs können heute nur von Spezialistinnen und Spezialisten (Infektiologen, Gastroenterologen oder Suchtmediziner mit Erfahrung in der Behandlung einer Hepatitis C) verschrieben werden. Da die Therapien aber unkompliziert sind, können Hausärzte über das Projekt HepCare (www.hepcare.ch) ihre Patientinnen und Patienten selber therapieren.
Beim Testen sollten wir, wie das Beispiel von L.F. zeigt, an die älteren Italienerinnen und Italiener denken, die in den 50er und 60er Jahren zu uns kamen. Erhebungen, die in den 1990er Jahren durchgeführt wurden, ergaben Prävalenzraten von bis zu zwölf Prozent für Hepatitis-C-Antikörper in einzelnen süditalienischen Dörfern [2]. An dieser Last trägt das Land bis heute: Der letzte Bericht des European Center for Disease Control schätzt die Prävalenz von Hepatitis C in der Allgemeinbevölkerung in Italien auf 3,9\% [7] und damit eine der höchsten in Europa (vgl. Abbildung «Hepatitis-C-Prävalenz in Europa»).

\section{Antikörpertest beim Check-up}

Neben den Italienerinnen und Italienern sind auch Einwanderer aus Spanien und Portugal und aus Balkanländern überdurchschnittlich häufig von Hepatitis $C$ betroffen [1]. Personen dieser Gruppen haben sich in vielen Fällen im medizinischen Bereich angesteckt. Als Hauptübertragungsrisiko in der übrigen Bevölkerung ist vor allem der intravenöse Konsum von Drogen zu nennen. Darunter sind auch Personen, die in der Vergangenheit kurz mit Drogen experimentiert und sich so angesteckt haben. Weiter gehören auch Bluttransfusionen und (zahn-)medizinische Eingriffe vor den 1990er Jahren zu den Risiken. Auch unhygienisch 
angebrachte Tattoos oder Piercings können zu Ansteckungen führen. Bei der allgemeinen Bevölkerung gibt es eine klare Altersverteilung bei den nachgewiesenen Infektionen: Die Geburtenkohorte 1950-1985 macht drei Viertel der Betroffenen aus [8].

Es lohnt sich also, zum Beispiel im Rahmen von Check-ups, einen HCV-Antikörpertest bei der Geburtenkohorte 1950-1985 oder bei Personen mit aktuellem oder Drogenkonsum in der Vergangenheit vorzuschlagen. Idealerweise wird beim Laborauftrag für die Antikörperbestimmung für den Fall eines positiven Resultates gleich eine HCV-RNA-Determinierung angefordert.

Wir sollten an die älteren Italienerinnen und Italiener denken, wenn es um Hepatitis C geht. Angesichts der einfachen Diagnosestellung und unkomplizierten Behandlung mit hohen Erfolgsaussichten ist es sinnvoll, dass sich Erstgenerationsmigranten aus Italien sowie die oben erwähnten Bevölkerungsgruppen mindestens einmal im Leben auf Hepatitis $C$ testen lassen, um sie im positiven Fall behandeln zu können.

\section{Die Schweizer Hepatitis-Strategie}

Der Verein Hepatitis Schweiz koordiniert die Schweizer HepatitisStrategie, ein Netzwerk von über 80 freiwilligen Expertinnen und Experten in der ganzen Schweiz, das Hepatitis B und C bis 2030 eliminieren möchte. Dieses Ziel hat auch die Weltgesundheitsorganisation WHO auf globaler Ebene gesetzt. Die Elimination ist realistisch: Eine wirksame Impfung schützt gegen eine Hepatitis-B-Infektion. Hepatitis C kann heute dank den effizienten Therapien fast immer geheilt werden. Nach wie vor sind jedoch zu viele Betroffene noch nicht diagnostiziert und behandelt. Um die Eliminationsziele zu erreichen, gilt es, mehr Menschen zu diagnostizieren und zu behandeln.

Weitere Informationen: www.hepatitis-schweiz.ch; www.hepcare.ch (Grundversorger behandeln Hepatitis C).
Literatur

1 Bertisch B, Giudici F, Negro F, Moradpour D, Müllhaupt B, Moriggia $\mathrm{A}$, et al. Characteristics of Foreign-Born Persons in the Swiss Hepatitis C Cohort Study: Implications for Screening Recommendations. PLOS ONE 11(5): e0155464.

2 Guadagnino V, Stroffolini T, Rapicetta M, Costantino A, Kondili LA, Menniti-Ippolito F, et al. Prevalence, risk factors, and genotype distribution of hepatitis $C$ virus infection in the general population: a community-based survey in southern Italy. Hepatology. 1997;26:1006-11.

3 Andriulli A, Stroffolini T, Mariano A, Valvano MR, Grattagliano, Ippolito AM, et al. Declining prevalence and increasing awareness of hepatitis $C$ virus infection in Italy: a population-based survey. Eur J Intern Med. 2018;53:79-84.

4 Kondili LA, Gamkrelidze I, Blach S, Marcellusi A, Galli M, Petta S, et al. Optimization of hepatitis $C$ virus screening strategies by birth cohort in Italy. Liver Int. 2020;40:1545-55.

5 Swiss Association for the Liver SASL, Schweizerische Gesellschaft für Gastroenterologie SGG und Schweizerische Gesellschaft für Infektiologie SGINF. Update Expert Opinion Statement: Treatment of Chronic Hepatitis C, Januar 2021.

6 Sarkar S, Jiang Z, Evon DM, Wahed AS, Hoofnagle JH. Fatigue before, during and after antiviral therapy of chronic hepatitis C: results from the Virahep-C study. J Hepatol. 2012;57(5):946-52.

7 ECDC Technical Report: Prevention of hepatitis B and C in the EU/ EEA and the UK, November 2020.

8 Bruggmann P, Richard J-L, on behalf of the Swiss Hepatitis C Cohort Study Group. Birth year distribution in reported hepatitis $C$ cases in Switzerland. European Journal of Public Health. 2015 (Feb); 25(1):141-3.

\section{Das Wichtigste in Kürze}

- In Italien geborene Menschen über 60, die in der Schweiz wohnen, fast vier Mal häufiger von Hepatitis $\mathrm{C}$ betroffen als der Rest der Schweizer Bevölkerung.

- Heute sollte allen chronisch mit Hepatitis C Infizierten eine Therapie angeboten werden.

- Heute sind Kombinationstherapien Standard, die ohne Interferon auskommen.

- Hausärztinnen, Hausärzte können über das Projekt HepCare (www.hepcare.ch) ihre Patientinnen und Patienten selber therapieren. 